EPJ Web of Conferences 41, 04029 (2013)

DOI: $10.1051 /$ epjconf/20134104029

(C) Owned by the authors, published by EDP Sciences, 2013

\title{
Decoherence in a Landau Quantized Two Dimensional Electron Gas
}

\author{
Jeremy A. Curtis ${ }^{1}$, Takahisa Tokumoto ${ }^{2}$, Judy Cherian ${ }^{2}$, Bagvanth Sangala ${ }^{1}$, Stephen A. McGill ${ }^{2}$, and \\ David J. Hilton ${ }^{1, \text { a }}$ \\ 1 University of Alabama, Birmingham, AL, USA \\ 2 National High Magnetic Field Laboratory, Florida State University, Tallahassee, FL, USA
}

\begin{abstract}
We have studied the dynamics of a high mobility two-dimensional electron gas as a function of temperature. The presence of satellite reflections in the sample and magnet can be modeled in the time-domain.
\end{abstract}

\section{Introduction}

We study a high-mobility $30 \mathrm{~nm} \mathrm{GaAs} / \mathrm{AlGaAs}$ two-dimensional electron gas using $\mathrm{THz}$ time domain spectroscopy (TTDS) with the sample in an applied magnetic field from $0.4 \mathrm{~K}-100 \mathrm{~K}$. In the presence of a perpendicular magnetic field, $\mathbf{B}$, the Fermi surface of the doped $\left(n=2 \times 10^{11} \mathrm{~cm}^{-2}\right)$ quantum well/two-dimensional electron gas splits into a spectrum of equally spaced Landau levels separated by the cyclotron energy, $\mathrm{h} v_{C R}$. An ultrafast terahertz pulse coherently couples the highest filled Landau level to the lowest unfilled level to create a coherent superposition between these states. Measurement of the transmitted $\mathrm{THz}$ pulse electric field has been previously shown to be a reliable, noncontact method to determine the effective mass, since $v_{C R}=e B /\left(2 \pi m^{*}\right)$ where $e$ is the electron charge and $m^{*}$ is the carrier effective mass, and can simultaneously determine the mobility, $\mu$, and carrier concentration, $n$, using the magnitude and lifetime of the induced cyclotron oscillations [1,2]. Our experimental work has extended this technique to lower temperatures where the sample dephasing lifetime/mobility increases due, in part, to the reduction in phonon population at low temperatures.

\section{Experiment}

Our $\mathrm{THz}$ time-domain spectrometer uses a $1 \mathrm{kHz}$ amplified titantium:sapphire laser that produces 150 fs pulses with $2 \mathrm{~mJ}$ per pulse. The THz time-domain spectrometer generated synchronized $\mathrm{THz}$ pulses using optical rectification in a (110) oriented ZnTe crystal. This experimental technique allows us to recover the full complex magnetoconductivity of our sample as a function of temperature and magnetic field in one measurement. As has been previously reported, this permits us to characterize the decoherence lifetime in external magnetic fields in the saturation limit [1,2], which has been difficult in the past using Fourier transform infrared (FTIR) (incoherent) based experiments.

\section{Results}

Representative data of the cyclotron oscillations at $0.4 \mathrm{~K}$ are shown in Figure 1(top). These show both the primary oscillation due to the initial transmission through the sample as well as the induced oscillations in a secondary reflection in the sample. In the highest quality samples, the narrow conductivity

\footnotetext{
a e-mail: dhilton@uab.edu
}

This is an Open Access article distributed under the terms of the Creative Commons Attribution License 2.0, which permits unrestricted use, distribution, and reproduction in any medium, provided the original work is properly cited. 

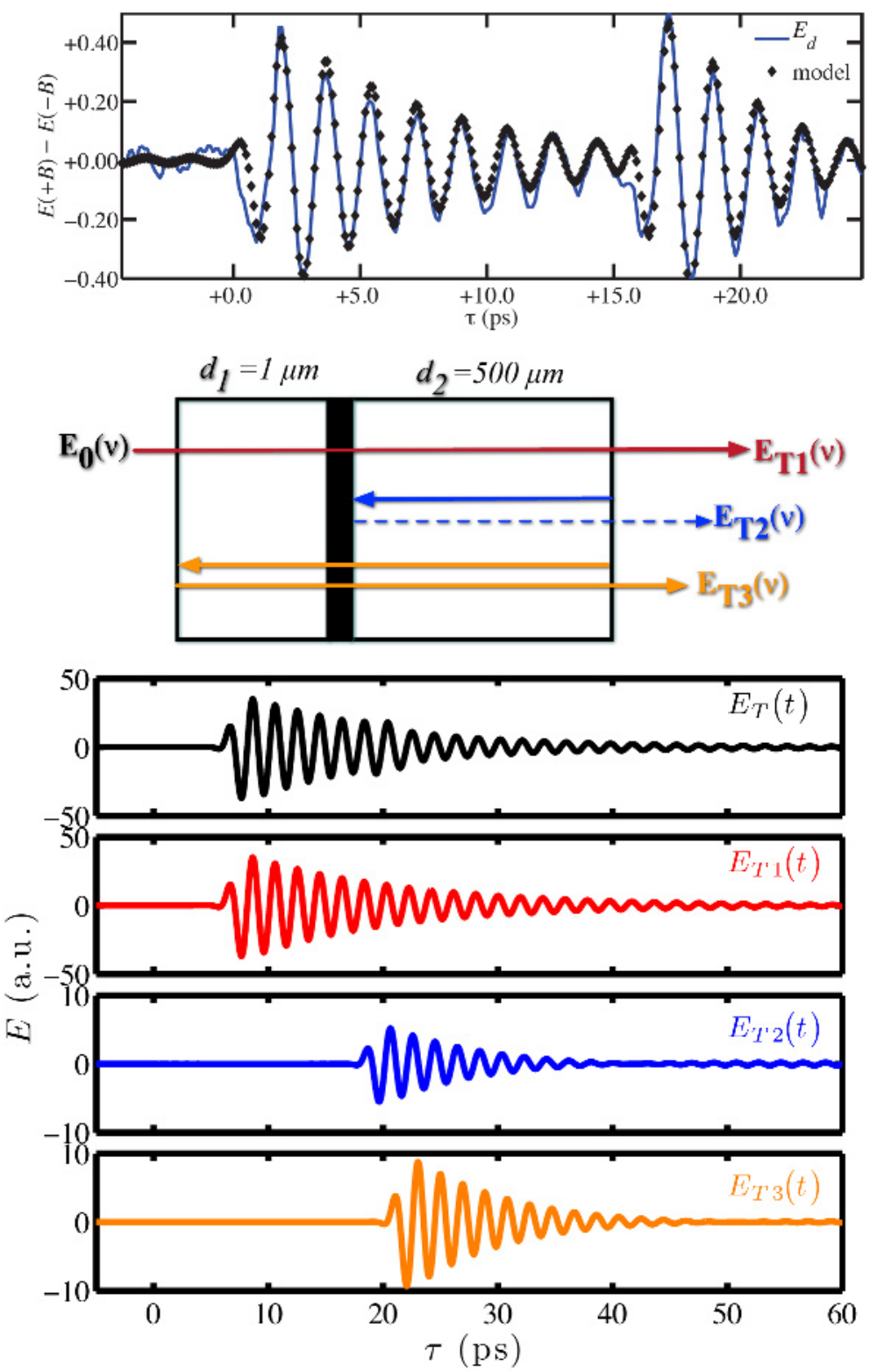

Fig. 1. (top) Sample cyclotron resonance data using $B=1.25 \mathrm{~T}$ and a GaAs $2 \mathrm{DEG}$ sample with a carrier concentration of $2 \times 10^{11} \mathrm{~cm}^{-2}$ and a mobility $3.4 \times 10^{6} \mathrm{~cm}^{2} \mathrm{~V}^{-1} \mathrm{~s}^{-1}$. (middle) A diagram showing the origin of the primary and first satellite pulses in our data. (bottom) A model of the transmitted waveform and its respective origins in the sample.

line width imposes an experimental limitation on the determination of cyclotron decoherence lifetimes. To properly analyze these high mobility samples, which have long decoherence lifetimes needed for future devices based on quantum state manipulation, new measurement and analysis techniques must be developed. In the magnet used in this experiment, optical windows and sample substrate result in the formation of a series of time-delayed satellite pulses located at times, $T_{i}$, after the initial pulse propagation through the sample. In our experiment, the first pulse occurs with a delay, $T_{A}$, of approx-

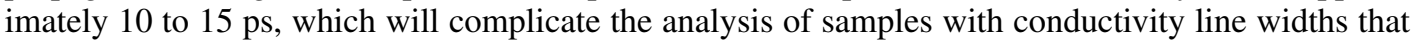


are narrower than $\sim 1 / 15 \mathrm{ps}=66 \mathrm{GHz}$, which corresponds to a mobility $\geq 4 \times 10^{5} \mathrm{~cm}^{2} \mathrm{~V}^{-1} \mathrm{~s}^{-1}$ in a GaAs based 2DEG. In those high mobility samples, cyclotron oscillations will persist and directly interfere with the secondary pulse transmission; this interference omits the use of standard temporal windowing techniques to isolate the initial response from any satellite pulses.

One solution would be to eliminate the satellite reflections using thicker or wedged substrates and windows, which is not a possible option in our particular experiment due to the specific configuration of this magnet. In this work, we use an analysis technique that relies on a direct fitting of multiple pulse reflections in the data to overcome the limitations of our experimental configuration. With no external magnetic field, the data show the multiple reflections that result from the windows and the sample substrate, while at finite field they additionally show a complex pattern of oscillations due to cyclotron motion in the 2DEG layer. These data can be modeled assuming that the only magnetically active layer is the $35 \mathrm{~nm}$ thick 2DEG layer that has a fixed sheet carrier concentration, $n=2 \times 10^{11} \mathrm{~cm}^{-2}$, and a Drude-like conductivity line shape centered at $v_{C R}$. With these assumptions, we can determine the temperature-dependent dephasing lifetime by fitting the multiple pulses and our known sample structure. To determine the layer thicknesses of the sample, substrate, and the set of windows in the data, we use the full waveforms transmitted through the sample to determine the optical path lengths, $n_{i} L_{i}$, of the layers with refractive index, $n_{i}$, and physical thickness, $L_{i}$. The initial satellite pulse results from a multiple reflection of the $\mathrm{THz}$ pulse in the substrate alone, while the subsequent pulses come from the windows, and higher order reflections from these primary sources. Figure 1(middle) shows a diagram of the origin of the single pass and initial satellite pulse, which demonstrates that the initial pulse transmits through the 2DEG layer a single time while the secondary pulse interacts with the 2DEG layer three times. To extract the decoherence lifetime, we fit these data to a time-domain model accounting for the time-delayed reflection pulses (see Figure 1(bottom) for a sample fitting), assuming a carrier concentration of $2 \times 10^{11} \mathrm{~cm}^{-2}$ and determining a decoherence lifetime of $\tau \approx 12.1 \mathrm{ps}$ from an initial best-fit of these data using a genetic algorithm minimization procedure. In this proceedure, we treat the waveform resulting from the interference of $E_{T 1}, E_{T 2}$, and, $E_{T 3}$ as a single pulse ariving at a time $T$ after the primary pulse. We model a single pulse with equation (1), where $u(t)$ is the heavyside step function, $\xi$ is the decoherence lifetime of the carriers (assumed to be equal for both pulses), and $t_{0}$ accounts for a possible shift in phase.

$$
g(t)=u(t) \exp \left(\frac{-t}{\xi}\right) \sin \left[2 \pi v_{c}\left(t+t_{0}\right)\right]
$$

The $y$-component of the complete transmitted field detected can be modeled using equation (2), where $A_{0}$ and $A_{1}$ are the amplitudes of the primary and secondary pulses, respectively. $\tau_{p}$ is the width of a gaussian we convolve $(\otimes)$ with the sum of the two pulses in order to account for the finite rise time of the carriers.

$$
\Delta E_{d, y}(t)=\left[A_{0} g(t)+A_{1} g(t+T)\right] \otimes \exp \left(\frac{-t^{2}}{\tau_{p}^{2}}\right)
$$

Further refinement of this analysis technique using higher order pulse reflections should result in a better estimate of the decoherence lifetime needed to replicate our time-domain data dynamics and amplitude [1,2].

\section{Acknowledgements}

This material is based upon work supported by the National Science Foundation under Grant No. DMR-1056827 and United States Department of Education under fellowship number P200A090143.

\section{References}

1. Wang X, Hilton DJ, Ren L, Mittleman DM, Kono J, Reno JL, Opt. Lett. 32, (2007) 1845-1847

2. Wang X, Hilton DJ, Reno JL, Mittleman DM, Kono J, Optics Express. 18, (2010) 12354-12361 